\title{
Excesive Scar Formation After Surgical Operations And Injuries: Expirience of Early Diagnosis and Prophyilaxis
}

\author{
Oxana Vladimirova ${ }^{1}$, Vladimir Vladimirov ${ }^{2}$, Karen Amlaev³ ${ }^{3}$ Peter Lavreshin ${ }^{4}$
}

\footnotetext{
1) General Surgery Department of Stavropol State Medical University, Stavropol / Russia

2) Oncologist of the Pyatigorsk Oncology Dispensary, Pyatigorsk / Russia

${ }^{3)}$ Head of Public Health Department of Stavropol State Medical University / Stavropol / Russia

${ }^{4)}$ Head of General Surgery Department of Stavropol State Medical University, Stavropol / Russia
}

\begin{abstract}
Aim: To show experience in determination of the risk factors of pathologic scars formations, in early diagnosis, to prove the importance of early prophylaxis and to provide the comparison in different prophylaxis schemes.

Material and Methods: According to our studies, more than $40 \%$ of patients in surgery and traumatology have an individual tendency to pathological scar formation, which must be taken into account when planning anti-scar therapy or prophylaxis. In addition, there are well-known factors that significantly increase the risk of formation of gross visible scars. Such as: Long-term wounds, inflammation, increased immune reaction of organism, technical specify of operation, strangulation of sutures, allergic reactions on threads and others.
\end{abstract}

Results: The result was monitored in each case for at least 6 months through clinical examination and dynamic examinations. Evaluation of the effectiveness was carried out by filling in the scar's card, including the subjective part - on the characteristics of the rumen on the basis of the patient's complaints, and objective - the data of the examination and research. Conclusion: For patients there are no small consequences of operations. Often there are the first things they ask is whether the scar remains. And our aim is to try make traces of surgical intervention as small as possible by easy, fast and effective way.

Keywords: Pathologic scars, prophylaxis, excesive scar

Vladimirova O., Vladimirov V., Amlaev K., Lavreshin P. Excesive Scar Formation After Surgical Operations And Injuries. Expirience of Early Diagnosis and Prophyilaxis. EJCM 2018; 06 (3): 107-110. Doi: 10.32596/ejcm.18.00307. 


\section{Introduction}

Relevance. Scar formation is problem as ancient as the existence of a human. Attitude to scars varied depending on fashion and century, but at all times the rough scars were like a mark of suffering and ugliness. The person who received such mark at all times understood that he could never get rid of it. But of course at all times people tried to eliminate scars. Nowadays the problem of scar formation continues to be one of the first in the system of rehabilitation treatment of patients after injuries and operations, which is apparently due to increased attention to the quality of life problems and the rapid restoration of working capacity.

Approximately 15 million surgical procedures performed in Russia per annum that may require scar prevention or therapy. From 5 to $16 \%$ patients in Russia develop pathological scars. Main problem is not in formation of scar itself, as it is physiological process as a part of wound healing, but in type of scar, that forming after operation. Scars classified on two main types: physiological and pathological. ${ }^{[1]}$ Our aim after operations is to reach the Normotrophic scar formation - the most physiological type of connective tissue substitution. Pathological scars divided on Hypertrophic scars, Keloids and Atrophic scars.

Patients with scars, especially keloid and hypertrophic, are very much concerned about the problem. Unpleasant persisting scars are significantly affecting patients psychologically, associate negative emotions after operation with scars, and feel insecure in their own skin regardless of operation success. The first problem for surgeons in this area is less information about tactics of early diagnostic and treatment. For example, there are some principal difference in prevention and treatment of keloid and hypertrophic scars. Keloid scars significantly protrude above the level of skin and is clear boundary, dense, sedentary, painful, often occur without previous trauma.

Keloids characterized by ability to constant growth and not ripen, i.e. collagen always remains young immature forms, as a result, the size of scar can be several times more than the wound size, not located, as a rule, in the joints region, do not form contractures. Such scars are very poorly treatable. Surgical excision is ineffective. At the same time, hypertrophic scars protrude above the surface of the skin, are limited to the injury area, do not occur without prior damage, respond well to treatment and prevention, may sometimes become smaller spontaneously, but often formed contractures and deformations of tissues.

There are many ambiguous approaches to the problem of hypertrophic scars. The effectiveness of most proposed methods of treatment and prevention are confirmed only by widespread use in practice over past years, while only few are tested in prospective studies with adequate control (the presence of reference control groups). For some of the technologies used, even safety data are not available, i.e. frequency of side effects. Some new approaches to scar therapy have shown good results in small trials, but have not been further investigated in large studies with an evaluation of longterm results. Evaluation of effectiveness is hampered by imperfect methods of quantitative measurement of dynamic changes in the appearance of scars, and their tendency to natural improvement over time. As a result, the current management of patients with scarring is based more on the personal experience of practicing physicians than on the results of large randomized controlled trials and other scientific evidence.

\section{Materials and methods}

According to our studies, ${ }^{[2]}$ more than $40 \%$ of patients in surgery and traumatology have an individual tendency to pathological scar formation, which must be taken into account when planning anti-scar therapy or prophylaxis. In addition, there are well-known factors that significantly increase the risk of formation of gross visible scars. Such as: Long-term wounds, inflammation, increased immune reaction of organism, technical specify of operation, strangulation of sutures, allergic reactions on threads and others.

Diagnosis of connective tissue hypertrophy is not very difficult and is based on anamnestic data and clinical manifestations. ${ }^{[2]}$ At the moment, there are a large number of diagnostic methods, that we used, including ultrasound, dermascopy and histological examination of wound or scar tissue, which make it possible to determine the type and dynamics of connective tissue growth. The fastest and easiest way to assess the degree of hyperplasia is ultrasound. The risk assessment is based on anamnestic data, features of the surgical wound or trauma and the timing of healing, the area of damaged tissues, the localization of the lesion, the 
anatomical and physiological properties of the skin, the detection of concomitant diseases, data on medications taken, the presence of antibodies in blood to scar antigens (author's technique), etc. For already formed scars and control the growth of scar tissue without use of hardware technology and histological examination, the Vancouver Universal Evaluation Scale, modified by $u^{2[2]}$, was used: thickness of tissues, thickness uniformity and vascularization of tissues, pigmentation of the injury zone, the consistence of the scar, itching, paresthesia and ulceration, scarring, and sensitivity.

There are two main directions in scars treatment: conservative methods and operational ones. ${ }^{[1,2,3]}$ And it is necessary to consider that later to start conservative treatment, more difficult it is to achieve significant effect. And surgical treatment is effective only at the late stages of scar maturation, not earlier than in a year, and is not suitable for all types of scars and all patients.

Moreover, most patients with scars who can be surgically treated simply refuse it for various reasons. And this problem can be solved by using specific original remedies in early stages of scar formation. As a rule, patients need to think about problem within first two years after its appearance, but this is time that most scars are needed for full maturation, and we shouldn't forget that to prevent always easier than to treat.

Since any scar tissue does not begin to form after wound healing, but much earlier, immediately after the inflammation phase, it is necessary to begin as soon as possible an adequate rehabilitation and specific prevention of pathological scar formation and prevent deterioration in the quality of patients life, as well as early recovery of work capacity, since the time factor and the complexity of the approach play an extremely important role in the effectiveness of the preventive measures being taken. In the surgical departments of the Clinical Hospitals of Stavropol Region for 11 years at different times were observed more than 300 patients aged 10 to 64 years of both sex with postoperative wounds and traumas. ${ }^{[2]}$ Knowing the fact that not in all people develop hypertrophic scars after damage to the skin and not everyone needs preventive treatment, we identified a predisposition for targeted prevention. It turned out that 212 patients, i.e. $69 \%$ of all had tendency to form excess connective tissue. All patients were divided into 3 groups. Group I, control, comprised 42 people who had no specific prophylaxis and received traditional treatment for postoperative wounds and applied elastic compression or silicone patches to the wound area beginning after the removal of the sutures.

In Group II, 114 patients were treated according to a standard regimen. After complete healing, a complex physiotherapy treatment was performed: magneto therapy, ultrasound on the scar with hormonal ointment, electrophoresis of the enzyme from the solution from the thirtieth day after the trauma and the duration of ten procedures per course. Most of the physiotherapy procedures were carried out already in the conditions of polyclinics. The course was repeated after 3 months. From 30 days after the operation, local compression or silicone pressing plasters were applied from 2 to 6 months for 8-14 hours a day. Local patients used nutritional cosmetics, there was no specific medical prevention of pathological scarring.

In the third group, consisting of 163 patients, early primary prevention of pathological scar formation in the early stages was carried out. All patients in the postoperative period were treated with modern wound coverings with protective, stimulating and antiseptic properties. From 3-7 days after the operation, a gel with an onion extract, which is a three-component preparation with a pronounced antihyperproliferative activity, was applied daily on zone around wound (not on the seam line in the presence of intradermal sutures to prevent loss of elasticity)

Since this antiplatelet drug consists of allantoin, heparin and an extract of onions, and only the original German preparation was used, which effectiveness and safety was proved by a large number of studies, side effects from the drug and contraindications in the form of hypersensitivity to parabens were not revealed. $[3,4]$ After removing the sutures, the gel application was continued by the patients themselves 2-3 times a day for 3 to 12 months. When applying the gel, compression bandages on the scar or silicone pressing plates were used, depending on the location of the scar. From 30 days after the operation, the course of physiotherapy began: ultraphoresis with gel with onion extract 10 procedures, laser, and electrophoresis with enzymes 10 procedures. Such courses repeated every 2-3 months.

\section{Results}

The result was monitored in each case for at least 6 months through clinical examination and dynamic ex- 
aminations. Evaluation of the effectiveness was carried out by filling in the scar's card, including the subjective part - on the characteristics of the rumen on the basis of the patient's complaints, and objective - the data of the examination and research.

\section{The obtained data were distributed as follows:}

In the group I, 32 patients developed hypertrophic scars requiring correction, later the patients did not follow the recommendations of the doctors and were excluded from the observation; 6 patients had pathological scars only from the cosmetic point of view, and in four the healing passed with the formation of the normotrophic scarring.

In the second group, observing during 6-18 months, the formation of pronounced hypertrophic scars in 45 patients was noted, while in the third group scar scarring was noted only in 19 patients. At the same time, 53 people from the 2 group noticed discomfort in the area of the formed scars, in the third group similar complaints were detected in 10 patients. The cosmetic defect was disturbed in the second group by 25 people, in the third group - 14. Functional disorders related to the development of scarring and tissue tension developed in group 2 in 39 patients, and in group 3 in 14 patients.

In addition, an assessment was made on the scales for individual signs of development of scars, where the best result was also obtained in group 3 of patients.

\section{Conclusions}

1. The problem of pathological scar formation, despite the rapid and progressive pace of development of modern medicine, remains extremely relevant for surgeons and traumatologists.

2. It was also concluded that primary prevention is necessary in all cases of predisposition to pathological scar formation, and taking into account the high frequency of predisposition and complexity, adjust scar at late stages of its maturation, recommend the prevention of scars after operations and injuries already at the stage of treatment of the underlying surgical disease.

3. The greatest effectiveness of complex therapy in the early stages of the formation of scar tissue with the use of modern wound coatings, early use of the original gel with onion extract locally in the form of applications and in ultraphonophoresis is shown.

4. The use of such a complex scheme significantly reduces the need for repeated surgical procedures, achieves a good cosmetic effect and largely eliminates such undesirable consequences of scar development as itching, feeling of tightening, decreasing of the full function of affected areas of the body where the scar is located, in the postoperative period, reduce the time for complete recovery and improve the quality of life of patients.

For patients there are no small consequences of operations. Often there are the first things they ask is whether the scar remains. And our aim is to try make traces of surgical intervention as small as possible by easy, fast and effective way.

\section{References}

1. G. Gauglitz, H. C. Korting, T. Pavicic, T. Ruzicka, and M. G. Jeschke, "Hypertrophic scarring and keloids: pathomechanisms and current and emerging treatment strategies," Molecular Medicine, vol. 17, no. 1-2, pp. $113-125,2011$

2. Vladimirova O.V. Complex approach to primary and secondary prevention of post-traumatic scars : diss. Stavropol; 2011. 135 p. (in Russian)

3. J. W. Cho, S. Y. Cho, S. R. Lee, and K. S. Lee, "Onion extract and quercetin induce matrix metalloproteinase-1 in vitro and in vivo," International Journal of Molecular Medicine, 2010;25(3): 347-352

4. K. Chanprapaph, S. Tanrattanakorn, P. Wattanakrai and co-authors. Effectiveness of Onion Extract Gel on Surgical Scars in Asians// Dermatology Research and Practice.Volume 2012 (2012)
Received: $28 / 05 / 2018$

Accepted: 10/09/2018

Published: 15/09/2018

Disclosure and conflicts of interest:

The authors declare no conflict of interest.

Corresponding author:

Oxana Vladimirova

Mail: oxy_8181@mail.ru 